J. Sustain. Wireless Syst.

Vol.01/ No. 01

Pages: $31-41$

http://irojournals.com/irosws/

DOI: https://doi.org/10.36548/jsws.2019.1.003

\title{
IOT BASED MULTI-HOMING APPLICATIONS - A REVIEW
}

\author{
N. Krishnaraj \\ Assistant Professor, Department of Information Technology, \\ Dr. Mahaligam College of Engineering and Technology, \\ Pollachi, India \\ E-mail: krishna.rajtce@gmail.com \\ S. Smys \\ Professor, Department of Computer Science and Engineering, \\ RVS Technical Campus, Coimbatore, India \\ E-mail: smys375@gmail.com
}

\begin{abstract}
With the ever increasing research in communication technology, smart systems and gadgets are emerging at a very fast pace. A recent trend is the research in internet of things (IoT) which facilitates access of information and services at any point of time around the globe thus elevating the process of digitization to a new era. Multi homing is a concept which facilitates connection of a user over multiple networks. These networks may be heterogeneous or homogeneous. This concept of multihoming acts as a strong backbone to wireless sensor networks where routing of information over the links and channels is quite a critical process. The routing of information from source to destination defines the overall accuracy of the entire wireless network. This efficiency largely dictated by an efficient routing process is influenced by many factors which include node efficiency, node lifetime, and the characteristics of the link between the nodes.
\end{abstract}

Keywords: Multihoming, IoT, Node lifetime, Node efficiency

\section{Introduction}

The concept of multi homing is understood based on the IP terminology, since it describes communication node or host as multi homed because it can be accessed by multiple IP address. 
J. Sustain. Wireless Syst.

Vol.01/ No. 01

Pages: $31-41$

http://irojournals.com/irosws/

DOI: https://doi.org/10.36548/jsws.2019.1.003

IP networks in the today world faces network failure and it needs a time to re-converge themselves to provide a new path. So routing plays a major role in multi homing in order to avoid the failure of paths in the network. Generally multihoming is designed with TCP Protocol and if provides resilience to the network failures and provides fast recovery with minimum time and during this time the traffic is considered as black holed within the network. Stream

controlled transmission protocol affects less by network re-convergence term since the packet losses are identified immediately and it retransmits through another address based on the routing also it is not affected by re-convergence. This can be implemented in the multiple interface networks to provide multipath communication through multiple IP address. Figure 1 illustrates a simple description about multi homing host. It consists of a single host and three interface lines such as N1, N2, N3 with different IP address to ensure the multi homed model and in the host there will be a unique transport number that uses IP address as IP1, IP2 and IP3.

HOST A

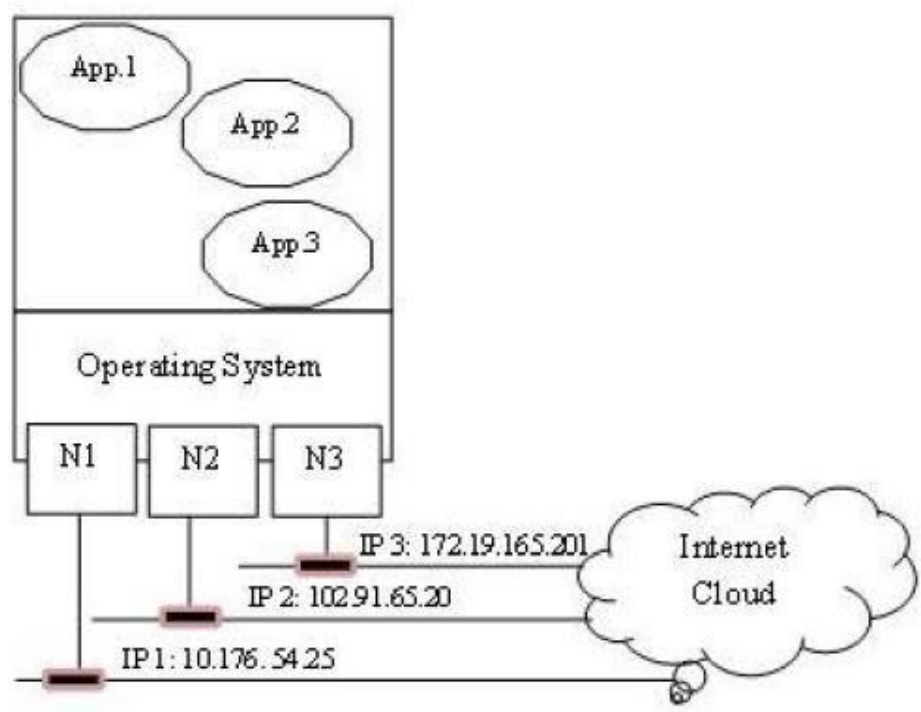

Figure 1 Multihomed host with multiple network interfaces

\section{Research Issues}

Commercial applications and various data transfer application needs a quality oriented 
J. Sustain. Wireless Syst.

Vol.01/ No. 01

Pages: $31-41$

http://irojournals.com/irosws/

DOI: https://doi.org/10.36548/jsws.2019.1.003

services to improve its robustness and the entire network performance. By varying the capacities and data utilization rates some applications does not satisfied with routing mechanism which is used in TCP and UDP since these layers are inadequate in performing large data. This motivates the development of SCTP in multi homing model along with the best routing protocol so that the performance of the system maintains its level and also increases the throughput. SCTP contains one or more building blocks called chunks for control the data at the time of congestion [1]. It uses a transmission sequence number for each protocol unit and also it provides acknowledgement like TCP. The motivation of multihoming in IoT is mainly based on the congestion control algorithms based on IEEE standard. This supports the multihoming model in transport layer by binding the IP address in the initial process itself. It allows multihomed [2] data transfer from multiple sources and destinations based on parameters such as retransmission rate. Concurrent multipath transfer performs a host to destination data transfer model for end to end communication and also it increases the network throughput. It maintains a receiver to receive buffer and it is shared across the various sub association flows [3] in case of path failure occurs.

\section{Methodology}

The path failure affects the performance of system since the minor delay in each failure results in to cumulative impact on file transfer thus it degrades the throughput of the network. The main objective of the thesis is to reduce the failure rates and improve the overall throughput. For achieving this a QoS [4] based transmission path is identified and this path is supports to carry redundant data in case of failure, until the path is recovered. This redundant path with redundant data acts as subsidiary path to overcome the path failure and reducing the packets drops. This dissertation introduces a new routing algorithm for multipath transmission in IoT which fulfils the needs and improves the overall performance.

Generally multi homing is integrated into concepts [5] such as multiple addressing, interfaces, overlay routing in the networks. Considering an example that multiple address are assigned to connect different terminals and the given network has prefixes on each connection 
J. Sustain. Wireless Syst.

Vol.01/ No. 01

Pages: $31-41$

http://irojournals.com/irosws/

DOI: https://doi.org/10.36548/jsws.2019.1.003

then there may be a problem of overlapping occurs in the address terminal. Since the multiple address has more similarities in the variables and less similarities in their variations. This over lapping concept in the network must be reconfigured over the entire network coverage area to sustain the user connectivity in the network. Overlay routing is basically integrated into a inter domain routing mechanism to increase the fault tolerance level of the model. This multi homing concept is simply classified into three categories such as [6]

1. End- Host Multi Homing

2. End- Site Multi Homing

3. Hybrid Multi Homing

Along with the multi homing concept internet of things is also integrated to introduce a new applications. This internet of things (IoT) [7] was first proposed by Kevin Ashton in 1999 by referring the interconnected objects in the radio frequency technology environment. Based on the applications the definition of internet of things (IoT) has many visions to provide solution for the many internet business groups for connecting many things into a single network. The definition for internet of things is given by European Research Cluster (ERC) as

"If a system has self-configuring capabilities based on the communication standards and its protocols in terms of physical or virtual for the dynamic global infrastructure network, then the things which are identical in the physical attributes and intelligent interference are integrated into a single informative network as Internet of Things (IoT)". 
J. Sustain. Wireless Syst.

Vol.01/ No. 01

Pages: $31-41$

http://irojournals.com/irosws/

DOI: https://doi.org/10.36548/jsws.2019.1.003

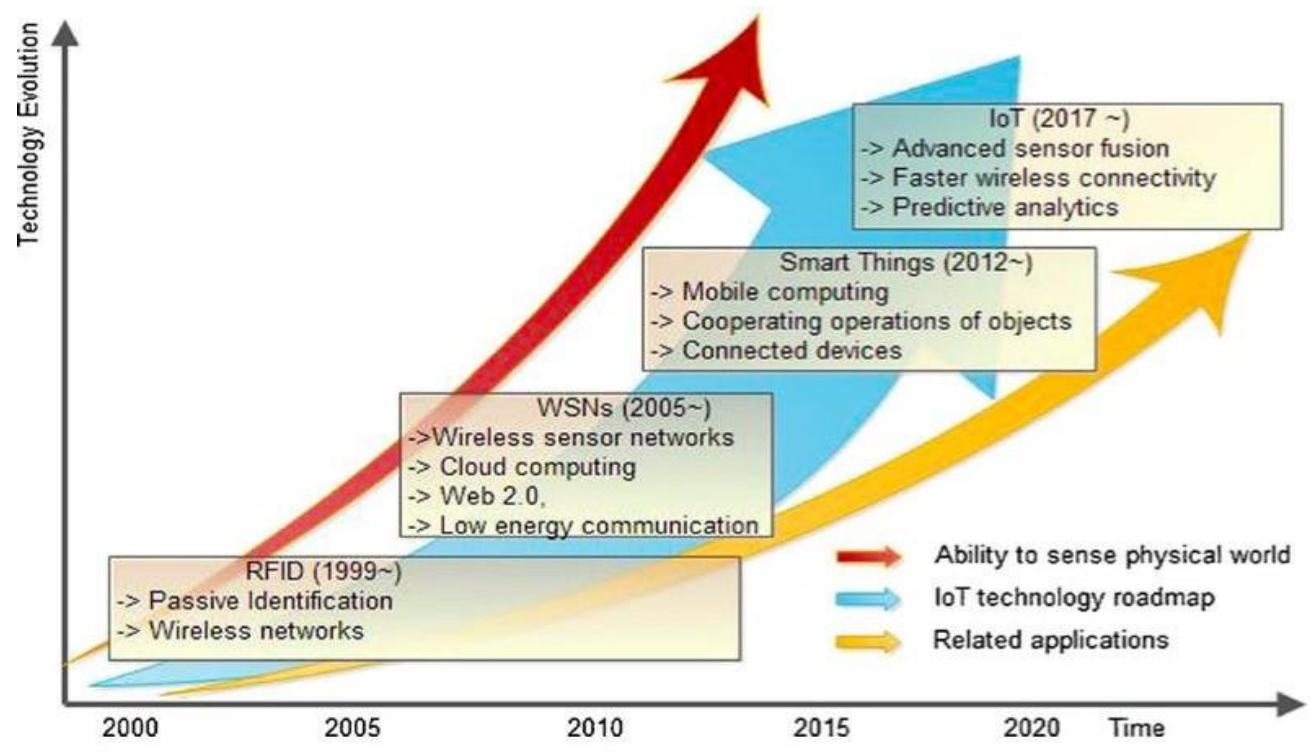

Figure 2. Growth of Internet of Things in present century

The growth of internet of things and its related researches are highlighted in the figure 2 . It starts from year 2000 and ends in 2020 [8] proposes the IoT applications has wide range in the future trends to enhance the advanced sensor fusion researches to improve the wireless connectivity. Figure 3 depicts an illustration of enterprise multi homing model includes ISP and Enterprise network which are interconnected through the links of internets. 
J. Sustain. Wireless Syst.

Vol.01/ No. 01

Pages: 31-41

http://irojournals.com/irosws/

DOI: https://doi.org/10.36548/jsws.2019.1.003

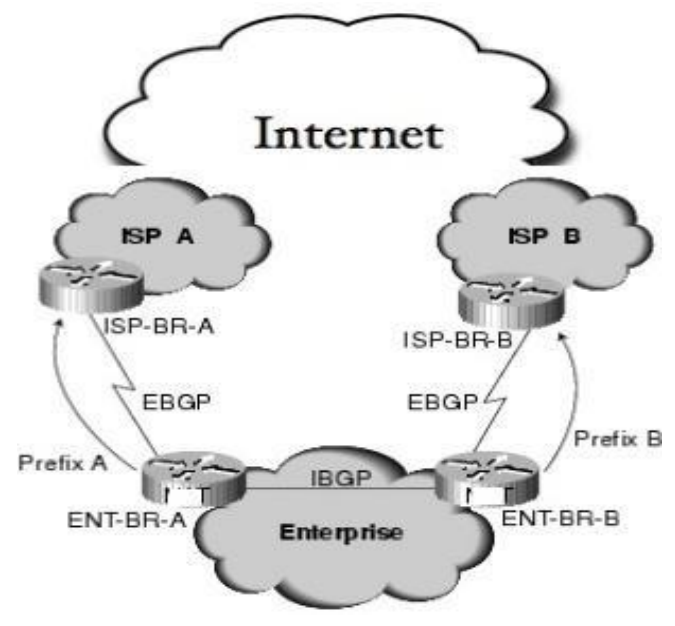

\section{Figure 3 Illustration of Enterprise Multi Homing}

End host Multihoming [9] is an entity configuration which includes several hop connection to the terminal end to select the own connection. Different logical or physical interfaces are used in the multi homed hose to interface the various networks and each network are configured by the service model. Prefix 1 and prefix 2 are the two interfaces used in the physical interface connections and it can be extended up to " $n$ " numbers [10].

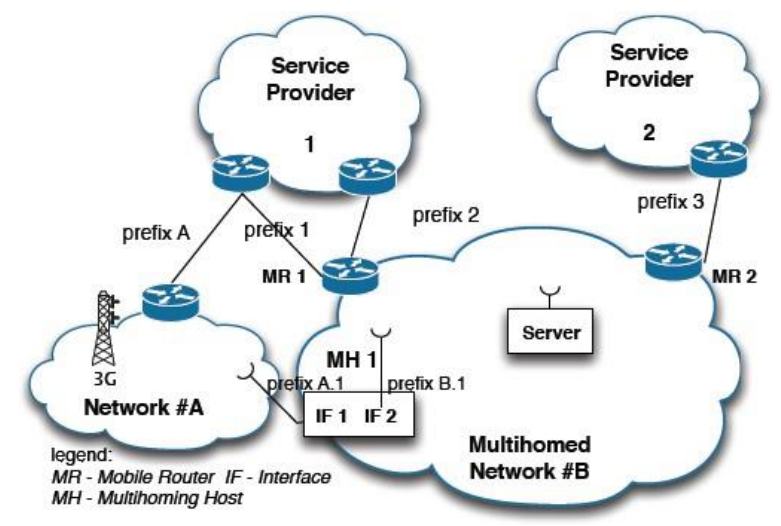

Figure 4. End Host Illustration- Multihoming Host 
J. Sustain. Wireless Syst.

Vol.01/ No. 01

Pages: $31-41$

http://irojournals.com/irosws/

DOI: https://doi.org/10.36548/jsws.2019.1.003

This multiple prefixes are used to configure the links and to access the network routers. Figure 4 is a descriptive picture about multihoming Host environment. From the figure it is observed that two service providers are used to connect in the main network which uses connecting lines as prefix 1, 2,3 for service provider 1 and 2. For network A it used prefix A as a connecting line to join in a multihomed network.

In the network configurations the first hop connections are given to the destinations to define the end site multihoming model. Based on the definitions for the end site multihoming multiple connections are used in the internet to increase the reliability of the network and also to improve the entire performance. This end site multi homing has redundant network connections which allows many networks for its services. The home agent and mobile routers are considered in the end site multihoming to provide internet access for the users in the network [11].

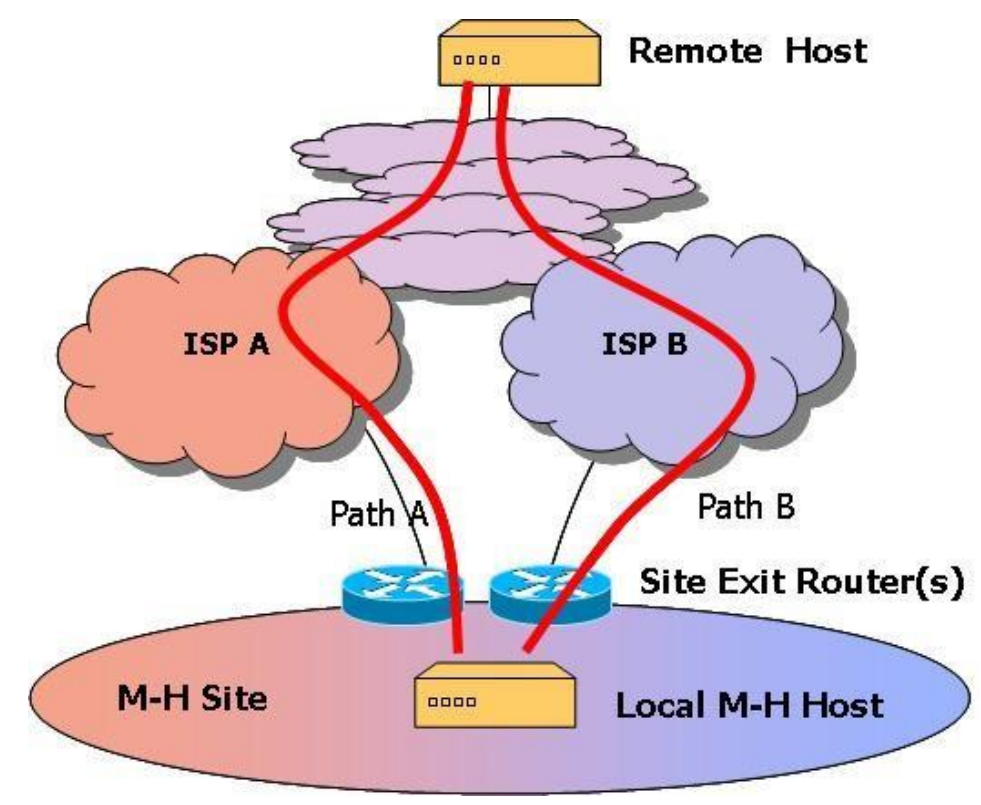


J. Sustain. Wireless Syst.

Vol.01/ No. 01

Pages: $31-41$

http://irojournals.com/irosws/

DOI: https://doi.org/10.36548/jsws.2019.1.003

\section{Figure 5. Illustration of End site modelling}

End site multihoming model process is simply based on the configuration and its problem orientation which has various parameters such as home agents, number of available prefixes and allocation of address in the internet service provided model. Figure 5 shows an illustration about the end site hosting model.

\section{HYBRID MULTIHOMING}

Hybrid multihoming broadly classified into three categories such as Content centric approach, Locator identifier split approach, Routing centric approach. In this the Content centric approach is used to perform routing based on the node and its interest in the network to exchange information. The architecture for service integration for supporting multihoming is added advantage in the content centric approach. In case of optimizing the future internet it incorporates the cross layer schemes for various functionalities. Service centric end to end abstraction approach has transfer of data from one node to nearest node to support flow distribution. Network named content mainly focuses on the data operations. If the node interested in sending packet types and $\mathrm{NNC}$ codes then based on the distinct table values the interest packets are transferred from one node to another node. These packets carry only about the information and not about the location. This hierarchical structure supports mobility and flow distribution [12]. 
J. Sustain. Wireless Syst.

Vol.01/ No. 01

Pages: $31-41$

http://irojournals.com/irosws/

DOI: https://doi.org/10.36548/jsws.2019.1.003

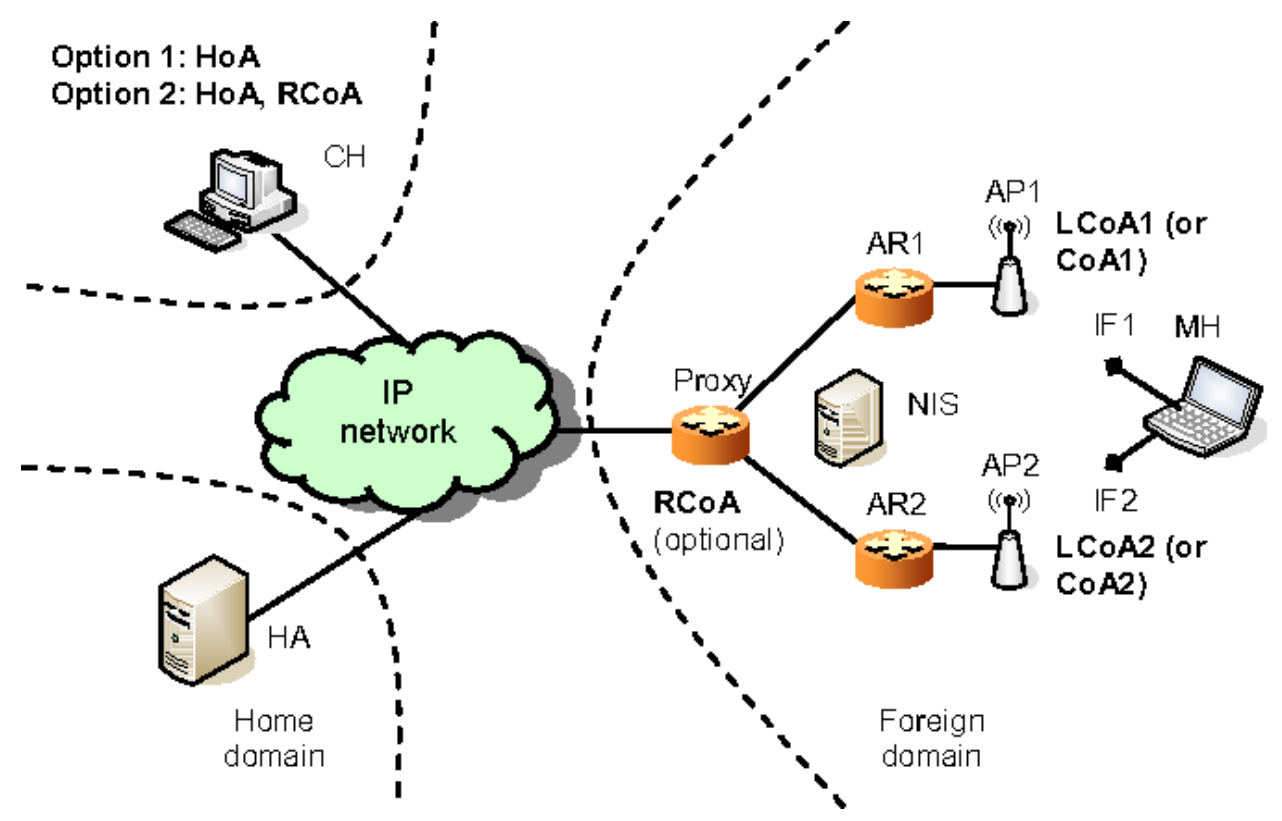

Figure 6. Hybrid Multihoming Model

Data oriented network architecture is used to generate key mechanism in the routing based client server process [13]. This method has reply for each client for finding the packets and the transport layer binds each name in the header and not the IP address in the TCP layer. This model assures the shortest path communication based on the confirmation from the server data and supports multiple connections since it has no public codes. Multiple access [14] information models allow information creation, distribution and retrieval among various nodes in the networks. It supports the local and global domain resources for name resolution services. This service has details about the applications and its domains and not about the details on information. Mobility and multi Homing support is an advantage in the multiple access information system in the present internet world and a real time example is a implementation in Firefox and thunderbird browsers [15].

\section{CONCLUSION}


J. Sustain. Wireless Syst.

Vol.01/ No. 01

Pages: $31-41$

http://irojournals.com/irosws/

DOI: https://doi.org/10.36548/jsws.2019.1.003

This research work describes about a brief description about multi homing and its associated research areas along with internet of things (IoT). Various network models and its types are analyzed in the first portion of chapter includes End- Host Multi Homing, End- Site Multi Homing, Hybrid Multi Homing. End host and end site multi homing models are analyzed based on the advantages and its limitations. Hybrid multi homing is discussed based on the content centric approach which provides a suitable mechanism to the multihoming environment. While considering multihoming mobility management and the research based results MIPv6 has suitable in the transport applications of multihoming along with this hybrid model.

\section{REFERENCES:}

[1] Satyam Agarwal; Swades De (2016), "Cognitive Multihoming System for Energy and Cost Aware Video Transmission" IEEE Transactions on Cognitive Communications and Networking, Vol. 2, No.3,pp.316-329.

[2] SelvakumarSasirekha; SankaranarayananSwamynathan (2017), "Cluster-chain mobile agent routing algorithm for efficient data aggregation in wireless sensor network" Journal of Communications and Networks, Vol.19, No.4, Pp. $392-401$

[3] SemiyeDemircan, Musa Aydin ,S. SavasDurduran (2011), "Finding optimum route of electrical energy transmission line using multi-criteria with Q-learning", Journal of Expert Systems with Applications- Elsevier,No.38,pp.3477-3482.

[4] ShahramJamali, Leila Rezaei, SajjadJahanbakhshGudakahriz (2013), "An Energyefficient Routing Protocol for MANETs: a Particle Swarm Optimization Approach" Journal of Applied Research and Technology, Vol.11, pp.803-812.

[5] SofianeHamrioui; Pascal Lorenz (2017), "Bio Inspired Routing Algorithm and Efficient Communications within IoT” IEEE Network, Vol.31, No.5, Pp. $74-79$

[6] Sun Baolin; Li Layuan (2004), "Optimizing on multiple constrained QoS multicast routing algorithms based on GA" Journal of Systems Engineering and Electronics, 
J. Sustain. Wireless Syst.

Vol.01/ No. 01

Pages: $31-41$

http://irojournals.com/irosws/

DOI: https://doi.org/10.36548/jsws.2019.1.003

Vol.15, No. 4, Pp. $677-683$

[7] Sun Baolin; Li Layuan (2005), "Multiple constraints-based QoS multicast routing: Model and algorithms" Journal of Systems Engineering and Electronics, Vol.16, No. 1, Pp. 187 $-193$

[8] Sungoh Kwon; Ness B. Shroff (2012), "Energy-Efficient Unified Routing Algorithm for Multi-Hop Wireless Networks" IEEE Transactions on Wireless Communications, Vol.11, No.11, Pp. 3890 - 3899

[9] SuvadipBatabyal; ParamaBhaumik (2015),"Mobility Models, Traces and Impact of Mobility on Opportunistic Routing Algorithms: A Survey" IEEE Communications Surveys \& Tutorials, Vol.17, No. 3, Pp. 1679 - 1707

[10] Swamy D. Ponpandi, AkhileshTyagi (2014), "User satisfaction aware routing and energy modeling of polymorphic network on chip architecture" Computers and Electrical Engineering, Vol.40, Pp.260-275.

[11] Romain Kuntz; Julien Montavont; Thomas Noel (2013), "Multihoming in IPv6 mobile networks: progress, challenges, and solutions", IEEE Communications Magazine,Vol.51,No.1, pp.128 - 135.

[12] Runzi Liu, Min Sheng, WeihuaWu(2018), "Energy-Efficient Resource Allocation for Heterogeneous Wireless Network With Multi-Homed User Equipments",Vol.6,No.1,Pp.14591-14601.

[13] SaiQian Zhang; Qi Zhang; HadiBannazadeh; Alberto Leon-Garcia (2015), "Routing Algorithms for Network Function Virtualization Enabled Multicast Topology on SDN", IEEE Transactions on Network and Service Management, Vol.12, No.4, Pp. 580 - 594

[14] SalimBitam; AbdelhamidMellouk; SheraliZeadally (2015) , "Bio-Inspired Routing Algorithms Survey for Vehicular Ad Hoc Networks"IEEE Communications Surveys \& Tutorials, Vol.17, No. 2, Pp. $843-867$

[15] Sandeep Kumar Singh; Tamal Das; AdmelaJukan (2015), “A Survey on Internet Multipath Routing and Provisioning" IEEE Communications Surveys \& Tutorials, Vol.17, No.4, Pp. $2157-2175$ 